\title{
TAME OBJECTS FOR FINITE COMMUTATIVE HOPF ALGEBRAS
}

\author{
WILLIAM C. WATERHOUSE
}

(Communicated by Louis J. Ratliff, Jr.)

\begin{abstract}
Let $S$ be an $A$-module algebra for a commutative Hopf algebra $A$, both projective of the same rank over a commutative ring. Let $I$ be the space of integrals in $A$. Then $S$ is an invertible $A$-module iff it is a faithful module which satisfies the "trace surjectivity" condition that 1 is in $\mathbf{I} S$.
\end{abstract}

Let $A$ be a commutative Hopf algebra, projective of rank $n$ over a commutative ring $R$. Let $S$ be a commutative algebra, also projective of rank $n$ over $R$, and suppose we have made $S$ an $A$-module algebra in the sense of Sweedler [4, p. 153]. That is, we have extended the $R$-module structure to an $A$-module structure that satisfies $b \cdot 1=\varepsilon(b) 1$ and $b \cdot s t=\sum\left(b_{i} \cdot s\right)\left(c_{i} \cdot t\right)$ for the counit $\varepsilon$ and the comultiplication $\Delta(b)=\sum b_{i} \otimes c_{i}$ in $A$. If $A$ is also cocommutative, this means that we have an action of the finite commutative group $\operatorname{scheme} \operatorname{Spec}\left(A^{*}\right)$ on $\operatorname{Spec}(S)$. Let $\mathbf{I}$ denote the set of integrals in $A$, so $\mathbf{I}=\{c \in A \mid b c=\varepsilon(b) c$ for all $b \in A\}$; this set is known to be a projective $R$-module of rank one [3, p. 592].

In a recent paper, Childs and Hurley [2] introduced some conditions on $S$ weaker than those making it a Galois object for $A$; they call it a tame object if (in addition to having the same rank as $A$ ) it satisfies

(i) $S$ is a faithful $A$-module,

(ii) $R=\{s \in S \mid b \cdot s=\varepsilon(b) s$ for all $b \in A\}$, and

(iii) $\mathbf{I} \cdot S=R$.

When $R$ is an integral domain and certain supplementary conditions are satisfied, Childs and Hurley showed that any tame object is actually an invertible $A$-module. (Taking their terminology from the case where $A$ is a commutative group algebra, they describe this by saying that $S$ has "local normal bases", reflecting the fact that when $R$ is local the ring $S$ will then-like $A^{*}$-be free over $A$.) In this note, I shall show that the conclusion actually holds in full generality for arbitrary $R$. Furthermore, condition (ii) can be dropped, and (i) can be weakened; the "trace surjectivity" condition (iii) is the crucial property.

THEOREM. Let $R$ be a commutative ring, $A$ a commutative Hopf algebra projective of rank $n$ over $R$. Let $\mathbf{I}$ be the integrals in $A$, and let $S$ be an A-module algebra also projective of rank $n$ over $R$. The following are equivalent:

(1) $S$ is tame in the sense of Childs and Hurley.

(2) $S_{Q} \neq 0$ for all $Q \in \operatorname{Spec}(A)$, and $1 \in \mathbf{I} \cdot S$.

(3) $S$ is an invertible $A$-module.

Received by the editors March 16, 1987.

1980 Mathematics Subject Classification (1985 Revision). Primary 14L20; Secondary 13B05.

This work was supported in part by the National Science Foundation, Grant MCS 8400649 . 
ProOF. As $S$ is finite over $A$, we know by $[1$, p. 133] that the primes $Q$ with $S_{Q} \neq 0$ are those containing the annihilator of $S$; thus (1) clearly implies (2).

Next we show that it suffices to prove $(2) \Rightarrow(3)$ and $(3) \Rightarrow(1)$ when $R$ is local. Let $P$ run through $\operatorname{Spec}(R)$. We know in advance that $S$ is finitely presented over $A$, since it is so over $R$, and hence by $[1$, p. 138] we see that condition (3) is true iff it is true over all localizations $R_{P}$. Similarly, as mentioned in [2, Corollary 2.6], each statement in the definition of tameness is true over $R$ iff it is true for each $R_{P}$. Finally, $\mathbf{I}_{P}$ is the set of integrals in $A_{P}$, and the primes of $A_{P}$ come from the primes of $A$ containing $P$, so condition (2) implies the corresponding conditions over all $R_{P}$.

Assuming $R$ local from now on, we show first that $(3) \Rightarrow(1)$. (Part of this is proved in [2, Theorem 2.7].) Since $R$ is local, $A$ is semilocal, and hence our invertible $S$ is actually free over $A$, say $S=A \cdot t$ for some $t$. Similarly, I is a free $R$-module, say $\mathbf{I}=R g$, and then $\mathbf{I} \cdot S$ is $R(g \cdot t)$. Obviously our $S$ is a faithful $A$-module. We can write $1=c \cdot t$ for some $c$ in $A$, and we have $b \cdot(c \cdot t)=b \cdot 1=\varepsilon(b) 1=\varepsilon(b) c \cdot t$ for all $b$. As $S$ is free, this equation implies $b \cdot c=\varepsilon(b) c$. Thus $c$ is in $\mathbf{I}$ and 1 is in $\mathbf{I} \cdot S$, so $R \subseteq \mathbf{I} \cdot S$. Similarly, any $s$ in $S$ that satisfies $b \cdot s=\varepsilon(b) s$ for all $b$ lies in I $\cdot S$, and thus we simply must show that $\mathbf{I} \cdot S$ is no bigger than $R$. But since $S$ is projective rank $n$ over $R$, it follows by $[1$, p. 176] that $R$ is a direct summand of $S$ as an $R$-module, say $S=R \oplus M$. Write $g \cdot t$ correspondingly as $r+m$. The element $c$ above is in $\mathbf{I}=R g$, say $c=r_{1} g$. Then $1+0=1=r_{1} g \cdot t=r_{1} r+r_{1} m$, which tells us first that $r_{1}$ is invertible and then that $m=0$. Thus indeed $\mathbf{I} \cdot S=R g \cdot t=R$.

Finally, still with $R$ local, we show that $(2) \Rightarrow(3)$. The first step is to show that $S$ is a projective $R$-module. This is essentially proved in [2, Theorem 5.1], so we just briefly sketch the argument here. The multiplication map $A \otimes S \rightarrow S$ is $A$-linear. As $R$ is local, $\mathbf{I}$ is free; let $g$ be a basis element for it, with (say) $\Delta g=\sum g_{i} \otimes h_{i}$. By assumption there is an element $u \in S$ with $g \cdot u=1$. Define a map $S \rightarrow A \otimes S$ by $x \mapsto \sum g_{i} \otimes u\left(s\left(h_{i}\right) \cdot x\right)$, where $s$ denotes the antipode in $A$. It is easy to verify that $\sum g_{i} \cdot u\left(s\left(h_{i}\right) \cdot x\right)=x$, so this map is a section of $A \otimes S \rightarrow S$, and a slightly longer computation (using the fact that $g$ is in $\mathbf{I}$ ) shows that the map is $A$-linear. Thus $S$ is an $A$-module direct summand of $A \otimes S$, which itself is automatically projective over $A$ since $S$ is so over $R$.

To complete the proof, note that $A$ is semilocal, and hence it can be decomposed as a finite product $A=A e_{1} \times \cdots \times A e_{r}$, where the $e_{i}$ are idempotents and $A e_{i}$ contains no nontrivial idempotents. Correspondingly then $S=e_{1} \cdot S \times \cdots \times e_{r} \cdot S$, and $e_{i} \cdot S$ is projective (and finitely generated) over $A e_{i}$. As $A e_{i}$ contains no nontrivial idempotents, each $e_{i} \cdot S$ has a well-defined rank $m_{i}$ over $A e_{i}$. Now each $A e_{i}$ is itself projective of some rank over the local ring $R$, and we have

$$
n=\operatorname{rank}_{R} S=\sum \operatorname{rank}_{R}\left(e_{i} \cdot S\right)=\sum m_{i}\left[\operatorname{rank}_{R}\left(A e_{i}\right)\right]
$$

But of course also

$$
n=\operatorname{rank}_{R} A=\sum \operatorname{rank}_{R}\left(A e_{i}\right) .
$$

Thus if any $m_{i}$ is greater than one, some other $m_{j}$ will have to be zero. But then we would have $S_{Q}=0$ for the primes $Q$ of $A$ not containing $e_{j}$, and this contradicts our hypothesis. Hence all $m_{j}$ are 1 , and $S$ has well-defined $\operatorname{rank} 1$ over $A$. As $A$ is semilocal, $S$ is actually free. 
COROLlARY. Let $R \rightarrow T$ be any ring homomorphism. If $S$ is tame for $A$ over $R$, then $S \otimes T$ is tame for $A \otimes T$ over $T$.

PROOF. This is not clear from the definition when $R \rightarrow T$, is not flat, but it is clear for the invertibility of the module. (The conditions in (2) also satisfy it.)

REMARK. If $A$ is not commutative, conditions (i), (ii), and (iii) do not imply the existence of local normal bases, not even in the basic case where $R=\mathbf{C}$ and $A$ is a group algebra $\mathbf{C}[G]$. Indeed, let $G$ for example be the symmetric group on three elements. The element $\sum_{G} \sigma$ is of course a basis for the integrals. Let $S$ be the algebra $\mathbf{C} \oplus M$, where $M$ is a 5-dimensional $\mathbf{C}$-space and $M^{2}=0$. To define a $\mathbf{C}[G]$ module algebra structure on $S$, we simply need to have $G$ acting on $S$ as algebra automorphisms. Define the action by using a decomposition $M=M_{1} \oplus M_{2}$, where $M_{1}$ is the 2-dimensional irreducible representation of $G$ and $M_{2}$ is a 3-dimensional space carrying three copies of the signum representation $\sigma \cdot m=\operatorname{sgn}(\sigma) m$. Clearly $S$ does not carry the regular representation of $\mathbf{C}[G]$, and thus there is no normal basis. But it is trivial to check that this $G$-action preserves the algebra structure, and furthermore in it;

(i) $S$ is a faithful $\mathbf{C}[G]$-module, as every type of irreducible representation occurs

(ii) the $s$ with $\sigma \cdot s=\varepsilon(\sigma) s$ for all $\sigma$ are the fixed elements, which by construction are just the elements in $\mathbf{C}$; and

(iii) $\mathbf{I} \cdot S=\left[\sum \sigma\right] \cdot S=\mathbf{C}$.

It would be quite interesting to find criteria for the existence of local normal bases for noncommutative $A$, particularly in the important (group scheme) case where $A$ is cocommutative.

\section{REFERENCES}

1. N. Bourbaki, Algèbre commutative, Chapitre 1, 2, Hermann, Paris, 1961.

2. L. N. Childs and S. Hurley, Tameness and local normal bases for objects of finite Hopf algebras, Trans. Amer. Math. Soc. 298 (1986), 763-778.

3. B. Pareigis, When Hopf algebras are Frobenius algebras, J. Algebra 18 (1971), 588-596.

4. M. E. Sweedler, Hopf algebras, Benjamin, New York, 1969.

Department of Mathematics, The Pennsylvania State University, UniverSITY PARK, PENNSYLVANiA 16802 